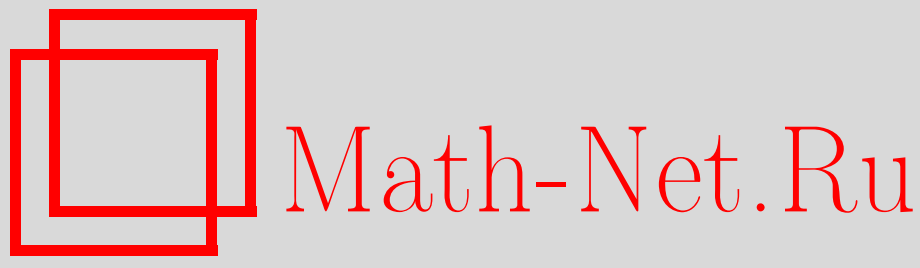

Обцероссийский математический портал

Л. А. Калякин, Усреднение в модели авторезонанса, Maтем. заметки, 2003, том 73, выпуск 3, 449-452

DOI: https://doi.org/10.4213/mzm615

Использование Общероссийского математического портала Math-Net.Ru подразумевает, что вы прочитали и согласны с пользовательским соглашением http://www . mathnet.ru/rus/agreement

Параметры загрузки:

IP : 54.197 .130 .99

26 апреля 2023 г., 16:33:29 


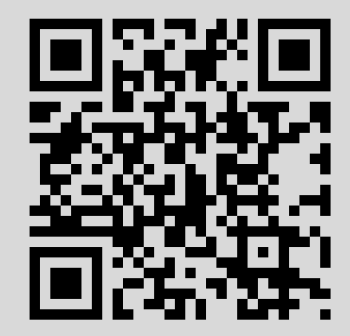




\section{УСРЕДНЕНИЕ В МОДЕЛИ АВТОРЕЗОНАНСА}

\section{Л. А. Калякин}

1. Постановка задачи. Рассматривается гамильтонова система двух дифференциальных уравнений

$$
u^{\prime}-H_{v}(u, v)=\varepsilon f(\tau) \cos (\varphi), \quad v^{\prime}+H_{u}(u, v)=\varepsilon g(\tau) \cos (\varphi), \quad u,\left.v(t ; \varepsilon)\right|_{t=0}=0,
$$

с малым множителем $\varepsilon$ при внешней вынуждающей силе, которая быстро осциллирует: $\varphi=\Phi(\tau) / \varepsilon$. Здесь $f, g, \Phi(\tau)$ - заданные гладкие функции медленного времени $\tau=\varepsilon t$. Предполагается, что невозмущенная система при $\varepsilon=0$ имеет в начале координат устойчивую неподвижную точку типа "центр" общего положения; эта точка берется в качестве начальной.

В данной заметке строится асимптотика решения $u, v(t ; \varepsilon)$ при $\varepsilon \rightarrow 0$, пригодная на далеких временах $t=\mathscr{O}\left(\varepsilon^{-1}\right)$. Построения выполняются с целью выявления условий возникновения авторезонанса. Это явление, аналогичное резонансу в линейной системе, характеризуется ростом энергии $H(u, v)+\varepsilon[v f-u g] \cos (\varphi)$ до величины порядка единицы при том, что вьнуждающая сила (накачка) мала: $\varepsilon f, \varepsilon g=o(1), \varepsilon \rightarrow 0,[1]-[3]$.

Конструкция основана на наличии у невозмущенной системы двухпараметрического семейства периодических по $t$ решений $u_{0}, v_{0}\left(t+t_{0}, E\right)\left(t_{0} \in \mathbb{R}, E \in\left(0, e_{0}\right) \subset \mathbb{R}\right)$ в окрестности неподвижной точки. Считается, что нулевое значение параметра $E$ соответствует точке равновесия: $u_{0}, v_{0}(t, 0) \equiv 0$. Этот параметр, как первый интеграл системы, можно отождествить с энергией: $E=H\left(u_{0}, v_{0}\right)$. Мы рассматриваем ситуацию общего положения, когда период невозмущенных (свободных) колебаний $T=T(E)$ и частота $\omega=2 \pi / T(E)$ зависят от энергии $E$, так что $\omega^{\prime}(E) \neq 0$.

Хотя по своей постановке задачавыглядит как двухчастотная с заданной внешней частотой $\Phi^{\prime}(\tau)$ и с собственной (свободной) частотой $\omega(E)$, нашей целью является построение одночастотного асимптотического решения. Выяснению необходимых условий сушествования такого решения посвящена эта заметка.

Основным исходным условием является требование совпадения частот в начальный момент $\Phi^{\prime}(0)=\omega(0)$. Если такое резонансное условие не выполнено, то асимптотическое решение остается мальм - порядка $\mathscr{O}(\varepsilon)$ на временах $t=\mathscr{O}\left(\varepsilon^{-1}\right)$ (т.е. фактически нелинейность оказывается

Работа выполнена при поддержке Российского фонда фундаментальных исследований, грант № 00-01-00663, программы "Ведущие научные школы", грант № 00-15-96038, и фонда INTAS, грант № 99-1068. 
слабой), в главньх членах асимптотики оно имеет двухчастотную структуру и строится методами типа ВКБ. Этот случай не имеет отношения к обсуждаемой нами проблеме авторезонанса.

Однако амплитуда вынужденных колебаний может оставаться малой даже при выполнении такого резонансного условия. Совпадение частот в начальный момент времени вовсе не гарантирует значительный рост амплитуды вынужденных колебаний. Так, при постоянной частоте накачки $\Phi^{\prime}(\tau) \equiv \omega(0)$ амплитуда нарастает до величины порядка $\mathscr{O}\left(\varepsilon^{1 / 3}\right)$ и не больше. Решение подобной задачи хорошо известно в связи с исследованиями явления синхронизации нелинейных колебаний, [4]. Невозможность дальнейшего роста амплитуды вынужденных колебаний объясняется зависимостью собственной частоты от энергии, из-за чего система со временем выходит из резонанса. Таким образом, для получения вынужденных колебаний с амплитудой порядка единицы за счет малоамплитудной накачки, необходимо медленное изменение вынуждающей частоты. Именно такой случай анализируется ниже.

Зависимость частоты свободных колебаний от энергии $\omega=\omega(E)$ представляет собой характерную черту нелинейных систем. Получаемое нами решение с растущей энергией $E=E(\tau)$ можно интерпретировать, как резултат автоматической подстройки нелинейной системы под внешние условия таким образом, что частота свободных колебаний $\omega(E(\tau))$ совпадает с частотой вынуждающей силы $\Phi^{\prime}(\tau)$. При некоторых условиях на накачку такой резонанс удерживается достаточно долго, и амплитуда колебаний (и энергия) нарастает до величины порядка единицы. Рассматриваемая задача инициирована рядом исследований из физики плазмы [5]-[7], [1]; последние достижения в этом направлении можно найти в публикациях по адресу http://socrates . berkeley.edu/ fajans/pub. Основное внимание обычно уделяется начальному этапу авторезонанса с использванием асимптотических приближений по малой амплитуде. Мы здесь анализируем этап далеких времен, когда амплитуда не мала.

2. Анзатц асимптотического решения. Для приближенного анализа применяется метод усреднения, который связывается с именами Крылова-Боголюбова-Кузмака [8]-[10] и которьй в физической литературе иногда ассоциируется с адиабатическими приближениями. В основе этого подхода лежит простое соображение, что главный член формального асимптотического решения $(\Phi A P)$ можно представить в виде решения невозмущенного уравнения

$$
\left(\begin{array}{l}
u \\
v
\end{array}\right)=\left(\begin{array}{l}
u_{0}(\sigma, E) \\
v_{0}(\sigma, E)
\end{array}\right)+\varepsilon\left(\begin{array}{l}
u_{1}(\sigma, \tau) \\
v_{1}(\sigma, \tau)
\end{array}\right)+\mathscr{O}\left(\varepsilon^{2}\right), \quad \tau=\varepsilon t
$$

при подходящем выборе фазы $\sigma=\varepsilon^{-1} \Psi(\tau ; \varepsilon) / \omega(E)$ и при подходящей деформации параметра $E=E(\tau, \varepsilon)$. В главном члене $\Phi$ АР задача сводится к нахождению функций $\Psi, E(\tau ; \varepsilon)$. Основной резултат работы составляет построение асимптотики этих медленно меняющихся функций

$$
\Psi(\tau ; \varepsilon)=\Psi_{0}(\tau)+\varepsilon \Psi_{1}(\tau)+\varepsilon^{2} \Psi_{2}(\tau)+\mathscr{O}\left(\varepsilon^{3}\right), \quad E(\tau, \varepsilon)=E_{0}(\tau)+\varepsilon E_{1}(\tau)+\mathscr{O}\left(\varepsilon^{2}\right) .
$$

3. Уравнения медленных деформаций. Класс функций $u, v(\sigma, \tau)$, в котором строится $\Phi А Р$, выделяется требованием $T$-периодичности по быстрой переменной $\sigma$. Условие периодичности поправок эквивалентно требованию отсутствия секулярных членов; оно приводит к уравнениям на медленные функции $\Psi, E$. Эти уравнения выписьваются в терминах невозмущенного решения и в главном члене асимптотики имеют вид

$$
\Psi_{0}^{\prime}=\omega\left(E_{0}\right), \quad E_{0}^{\prime}=\frac{1}{T} \int_{0}^{T}\left[\partial_{\sigma} \mathbf{u}_{0}\left(\sigma, E_{0}\right), \mathbf{F}(\varphi ; \tau)\right] d \sigma
$$

Выражение под интегралом представляет собой кососкалярное произведение производной вектора невозмущенного решения $\mathbf{u}_{0}=\left(u_{0}, v_{0}\right)$ и вектора возмущения $\mathbf{F}=(f, g) \cos (\varphi)$.

Следует учесть, что исходные уравнения уже содержат в явной форме зависимость от быстрой переменной $\varphi=\Phi(\tau) / \varepsilon$ с заданной функцией $\Phi(\tau)$. В интересующем нас случае авторезонанса можно догадаться, что быстрые переменные, заданная $\varphi$ и искомая $\sigma$ должны быть связаны. Такая связь формулируется в следующей форме. 
4. Резонансное требование. Разность между собственной и вынуждающ,ей частотами мала $\Psi^{\prime}-\Phi^{\prime}=\mathscr{O}(\varepsilon)$.

Это соотношение является дополнительным к анзатцу. Попросту говоря, в качестве главного члена быстрой фазы берется фаза накачки $\Psi_{0}=\Phi(\tau)$, и требуется найти сдвиг фазы вынужденных колебаний. В таком подходе первое уравнение в (4) оказьвается алгебраическим уравнением $\omega\left(E_{0}\right)=\Phi^{\prime}(\tau)$ для энергии $E_{0}$. После этого второе уравнение превращается в алгебраическое уравнение для главного члена сдвига фазы $\Psi_{1}$, если учесть связь быстрых переменных, задаваемую резонансньм требованием: $\varphi=\omega(E) \sigma-(\Psi-\Phi) / \varepsilon$. Из анализа последующих приближений нетрудно выписать линейные алгебраические уравнения для поправок в асимптотике функций $E, \Psi$.

5. Результаты. Ясно, что в общем случае нелинейные алгебраические уравнения (4) не разрешимы. Условия их разрешимости являются необходимыми для реализации описанного подхода и интерпретируются, как условия возникновения авторезонанса.

Первое уравнение в (4) соответствует требованию совпадения собственной частоты с частотой накачки. Из него определяется главньй член асимптотики для медленно меняющейся энергии $E_{0}=E_{0}(\tau)$. Условие разрешимости выделяет класс вынуждающих частот, при которых только и возможен авторезонанс.

TEOPEмA 1. Пусть частота накачки в начальный момент совпадает с собственной $\Phi^{\prime}(0)=\omega(0) u \omega^{\prime}(E) \neq 0$. Первое уравнение в (4) имеет единственное растущее решение $E_{0}=E_{0}(\tau)$ на промехутке $\tau \in\left[0, \tau_{0}\right]$ тогда и только тогда, когда совпадают направления изменения частот $\operatorname{sgn} \Phi^{\prime \prime}(\tau)=\operatorname{sgn} \omega^{\prime}(E)$. В качестве $E_{0}(\tau)$ берется корень со свойством $E_{0}(0)=0$.

Второе уравнение в (4) можно привести к виду

$$
-\frac{\omega\left(E_{0}\right)}{2 \pi} \int_{0}^{2 \pi}\left[\mathbf{u}_{0}\left(\frac{s}{\omega\left(E_{0}\right)}\right), \partial_{s} \mathbf{F}\left(s-\Psi_{1} ; \tau\right)\right] d s=\frac{\Phi^{\prime \prime}(\tau)}{\omega^{\prime}\left(E_{0}\right)}
$$

Разрешимость этого уравнения относительно $\Psi_{1}=\Psi_{1}(\tau)$ возможна при определенных ограничениях на исходные данные накачки $\mathbf{F}(s, \tau)$ и $\Phi(\tau)$. В частности, из уравнения (5) вытекает, что скорость изменения частоты накачки в начальный момент должна быть равна нулю $\Phi^{\prime \prime}(0)=0$, поскольку амплитуда невозмущенного решения равна нулю при нулевой энергии $\mathbf{u}_{0}\left(T\left(E_{0}\right) s / 2 \pi\right.$, $\left.E_{0}\right) \rightarrow 0, E_{0}(\tau) \rightarrow 0$ при $\tau \rightarrow 0$.

Наиболее простая ситуация возникает в случае, когда вектор накачки имеет одну гармонику, как в уравнениях (1). Тогда задача для $\Psi_{1}$ сводится к тригонометрическому уравнению

$$
\Phi^{\prime}(\tau)\left[a(\tau) \cos \left(\Psi_{1}\right)-b(\tau) \sin \left(\Psi_{1}\right)\right]=\frac{\Phi^{\prime \prime}(\tau)}{\omega^{\prime}\left(E_{0}(\tau)\right)} .
$$

В нем участвуют два коэффициента Фурье от комбинации компонент невозмущенного решения

$$
a=\frac{1}{2 \pi} \int_{0}^{2 \pi}\left[g(\tau) u_{0}-f(\tau) v_{0}\right] \sin (s) d s, \quad b=\frac{1}{2 \pi} \int_{0}^{2 \pi}\left[g(\tau) u_{0}-f(\tau) v_{0}\right] \cos (s) d s .
$$

Эти коэффициенты известны настолько, насколько известно невозмущенное решение $\left(u_{0}, v_{0}\right)(\sigma, E)$, взятое при $\sigma=T(E) s / 2 \pi$ и $E=E_{0}(\tau)$.

Теорема 2. Пусть правая часть в исходной задаче задана в форме (1) и выполнены условия теоремы 1. Уравнение (6) разрешимо тогда и только тогда, когда амплитуда и фаза связаны неравенством

$$
a^{2}(\tau)+b^{2}(\tau) \geqslant\left[\frac{\Phi^{\prime \prime}(\tau)}{\omega \omega^{\prime}\left(E_{0}(\tau)\right)}\right]^{2} .
$$

Если уравнение (6) разрешимо, то существуют два и только два решения $\Psi_{1}=\Psi_{1}^{ \pm}(\tau)$. Корни различны $\Psi_{1}^{+}(\tau) \neq \Psi_{1}^{-}(\tau)$, если неравенство $(7)$ - строгое.

Таким образом, для возникновения авторезонанса необходимо превьшение амплитуды накачки над некоторым пороговым значением. Если для невозмущенного решения использовать асимптотику по малой энергии, то пороговое условие можно упростить. 
СлЕДСтвИЕ 2.1. Для разрешимости уравнения (6) в окрестности начальной точки $\tau=0$ достаточно выполнения строгого неравенства в начальный момент:

$$
\omega^{2}(0)\left[f^{2}(0)+g^{2}(0)\right]>4\left|\frac{\Phi^{\prime \prime \prime}(0)}{\omega^{\prime}(0)}\right|
$$

$n p u \Phi^{\prime \prime}(0)=0$.

Вывод. Условия разрешимости уравнений (4) можно интерпретировать, как необходимые условия возникновения авторезонанса, поскольку на построенном таким образом ФАР (2), (3) энергия нарастает до величинь порядка единицы. Те же условия обеспечивают реверс с убьванием энергии при соответствующем изменении частоты накачки.

Асимптотический анализ подтверждается численными экспериментами на примере уравнения с кубической нелинейностью.

6. Обсуждение результатов. В главном члене $Ф А Р$ имеется неоднозначность из-за произвола в выборе корня для сдвига фазы $\Psi_{1}$. Старшие члены $\Phi$ АР $(2),(3)$ находятся из линейных уравнений, и дополнительных ограничений на исходные данные не возникает. Однако в старших коэффициентах асимптотики медленных функций обнаруживаются особенности при $\tau \rightarrow 0$; например, $E_{1}(\tau)=\mathscr{O}\left(\tau^{-1}\right), \Psi_{2}(\tau)=\mathscr{O}\left(\tau^{-3}\right)$. При этом порядок сингулярности нарастает с ростом номера приближения. Такие особенности свидетельствуют о непригодности $\Phi A P(2),(3)$ на начальном этапе. Область пригодности описьвается неравенством $\varepsilon^{-2 / 3} \ll t \leqslant \mathscr{O}\left(\varepsilon^{-1}\right)$. Асимптотическое приближение на начальном этапе следует строить в другой форме с использованием другого медленного времени $\theta=\varepsilon^{2 / 3} t,[11]$. Такая структура решения напоминает погранслой. Так же, как в похожих задачах с бисингулярным возмущением [12], согласование двух ФАР обеспечивает ликвидацию всех неоднозначностей во "внешнем" $\Phi А Р(2),(3)$ и, в частности, обеспечивает однозначньй выбор сдвига фазы $\Psi_{1}$. Вопрос о влиянии начальных данных на возникновение авторезонанса решается при исследовании погранслойной задачи и выходит за рамки данной заметки.

\section{СПИСОК ЦИТИРОВАННОЙ ЛИТЕРАТУРЫ}

1. Голованевский К. С. // Физика плазмы. 1985. Т. 11. № 3. С. 295-299. 2. Friedland L. // Phys. Rev. E. 1997. V. 55. P. 1929-1939. 3. Friedland L. // Phys. Rev. E. 2000. V. 61. P. 3732-3735. 4. Андронов А. А., Витт А. А., Хайкин С. Э. Теория колебаний. М.: Наука, 1981. 5. Векслер В.И. // Докл. АН СССР. 1944. Т. 43. № 8. С. 346-348. 6. Векслер В.И. // Докл. АН СССР. 1944. Т. 44. № 9. С. 393-396. 7. Андронов А. А., Горелик Г. А. // Докл. АН СССР. 1945. Т. 49. №9. С. 664-666. 8. Крылов Н. М., Боголюбов Н. Н. Исследование продольной устойчивости аэроплана. М.-Л.: Гос. авиац. и автотракт. изд-во, 1932. 9. Кузмак Г. Е. // Изв. AH CCCP. Cep. матем. 1959. T. 23. № 3. C. 515-526. 10. Bourland F. J., Haberman R. // SIAM J. Appl. Math. 1988. V. 48. № 4. Р. 737-748. 11. Калякин Л. А. // Докл. РАН. 2001. Т. 378. № 5. С. 594-597. 12. Ильин А. М. Согласование асимптотических разложений решений краевых задач. М.: Наука, 1989. 